\title{
A HIGH-ORDER SPECTRAL METHOD FOR NONLINEAR WATER WAVES OVER MOVING BOTTOM TOPOGRAPHY*
}

\author{
PHILIPPE GUYENNE ${ }^{\dagger}$ AND DAVID P. NICHOLLS ${ }^{\ddagger}$
}

\begin{abstract}
We present a numerical method for simulations of nonlinear surface water waves over variable bathymetry. It is applicable to either two- or three-dimensional flows, as well as to either static or moving bottom topography. The method is based on the reduction of the problem to a lower-dimensional Hamiltonian system involving boundary quantities alone. A key component of this formulation is the Dirichlet-Neumann operator which, in light of its joint analyticity properties with respect to surface and bottom deformations, is computed using its Taylor series representation. We present new, stabilized forms for the Taylor terms, each of which is efficiently computed by a pseudospectral method using the fast Fourier transform. Physically relevant applications are displayed to illustrate the performance of the method; comparisons with analytical solutions and laboratory experiments are provided.
\end{abstract}

Key words. gravity water waves, bathymetry, pseudospectral methods, Dirichlet-Neumann operators, boundary perturbations

AMS subject classifications. 76B15, 76B07, 65M70, 35S30

DOI. $10.1137 / 060666214$

1. Introduction. Accurate modeling of surface water wave dynamics over bottom topography is of great importance to coastal engineers and has drawn considerable attention in recent years. Here are just a few applications: linear [16, 33] and nonlinear wave shoaling [25, 24, 28, 27], Bragg reflection [35, 15, 36, 14, 30, 33, 4], harmonic wave generation $[3,17,18]$, and tsunami generation $[37,22]$. As the publications cited above attest, the character of coastal wave dynamics can be very complex: when entering shallow water, waves are strongly affected by the bottom through shoaling, refraction, diffraction, and reflection. Nonlinear effects related to wave-wave and wave-bottom interactions can cause wave scattering and depth-induced wave breaking. In turn, the resulting nonlinear waves can have a great influence on sediment transport and the formation of sandbars in nearshore regions. The presence of bottom topography also introduces additional space and time scales to the classical perturbation problem (see, e.g., [10]).

Traditionally, the water wave problem on variable depth has been modeled using long-wave approximations such as the Boussinesq or shallow water equations [26, 40]. See also [39] for a weakly nonlinear formulation for water waves over topography. In recent years, progress in both mathematical techniques and computer power has led to a rapid development of numerical models solving the full Euler equations. These can be divided into two main categories: boundary integral methods $[2,24,49,7,20,27]$ and spectral methods. In particular, efficient spectral methods based on perturbation expansions have been developed for the computation of water waves on constant or

*Received by the editors July 27, 2006; accepted for publication (in revised form) April 20, 2007; published electronically November 16, 2007.

http://www.siam.org/journals/sisc/30-1/66621.html

$\dagger$ Department of Mathematical Sciences, University of Delaware, Newark, DE 19716 (guyenne@ math.udel.edu). This author's work was supported by the University of Delaware Research Foundation and the NSF through grant DMS-0625931.

${ }^{\ddagger}$ Department of Mathematics, Statistics, and Computer Science, University of Illinois at Chicago, Chicago, IL 60607 (nicholls@math.uic.edu). This author's work was supported by the NSF through grant DMS-0537511. 
infinite depth $[19,48,13,41,1]$, and related methods have been proposed to include the effects of an uneven bottom [33, 46, 4, 28].

In this paper, we present a new numerical method for studying nonlinear water waves on variable depth which extends the work of Craig and Sulem [13] and Guyenne and Nicholls [28] in several important directions. As in the work listed above, the method is based upon Zakharov's Hamiltonian formulation [50], which was further refined by Craig and Sulem [13] and Craig et al. [10]. In this formulation the DirichletNeumann operator (which produces the normal fluid velocity at the water surface given the surface shape and the velocity potential there) plays a central role. Following previous work in the case of trivial bathymetry $[8,12,11,42,44]$, it was recently shown [45] that this Dirichlet-Neumann operator (DNO) is jointly analytic with respect to deformations of both the trivial surface (quiescent water) and trivial topography (flat bottom). Furthermore, the (pseudodifferential) terms in the (double) Taylor series of the DNO can be formally calculated $[46,10]$ and, after truncation at a certain order, used to simulate the action of the DNO. Due to the pseudodifferential character of the operators involved, the spatial discretization of our problem is efficiently performed by a Fourier pseudospectral method using the fast Fourier transform.

Our novel contributions are two-fold: First, the formal expressions for the Taylor series terms of the DNO have been rewritten to eliminate the explicit appearance of extremely unstable operators which, in a sense made clear in section 2 , are "exponentially unbounded." Second, our generalized formulation of the problem now allows for moving bottom topography so that we may, for example, simulate the sudden motion of the bathymetry as in the case of an earthquake-generated tsunami. Finally, we provide five physically motivated problems from the field of coastal engineering which are, for the first time, simulated with a high-order spectral, boundary perturbation scheme.

The paper is organized as follows: In section 2, we present the mathematical formulation of the problem and derive Taylor series expansions of the DNO for both static and moving bottom topography. In section 3, we give a description of the numerical methods for discretization of the equations of motion and for wave generation/absorption in the model. Finally, we perform a series of experiments to demonstrate the effectiveness of the model in section 4 . We make some concluding remarks in section 5 .

2. Mathematical formulation. We consider the motion of a free surface, $\eta(x, t)$, on top of a fluid domain defined by

$$
S(\beta, \eta)=\left\{(x, y) \in \mathbb{R}^{n-1} \times \mathbb{R} \mid-h_{0}+\beta(x, t)<y<\eta(x, t)\right\},
$$

where $\beta(x, t)$ denotes the bottom perturbation and $n$ is the spatial dimension. The quiescent water level is located at $y=0$ and the constant reference depth is $h_{0}$. We assume the fluid is incompressible and inviscid, and the flow is irrotational, so that the fluid velocity can be expressed as $u=\nabla \varphi$, where $\varphi$ denotes the velocity potential. Under the above assumptions, the full boundary value problem for potential flow is given by

$$
\begin{aligned}
\Delta \varphi=0 & \text { in } S(\beta, \eta), \\
\partial_{t} \eta+\nabla_{x} \varphi \cdot \nabla_{x} \eta-\partial_{y} \varphi=0 & \text { at } y=\eta(x, t), \\
\partial_{t} \varphi+\frac{1}{2}|\nabla \varphi|^{2}+g \eta=0 & \text { at } y=\eta(x, t), \\
\nabla \varphi \cdot \nu(\beta)=v(x, t) & \text { at } y=-h(x, t)
\end{aligned}
$$


(see [31]), where $g$ is the acceleration due to gravity, $\nu(\beta)=\left(-\nabla_{x} \beta, 1\right)$ is a (nonnormalized) upward vector normal to the bottom, and $h(x, t)=h_{0}-\beta(x, t)$ is the fluid depth. Surface tension effects are neglected but could easily be included in (2.1c) (see, e.g., [11]). The bottom motion is determined by the normal velocity $v(x, t)$ which vanishes in the case of a fixed bottom.

Following Craig and Sulem [13], we can reduce the dimensionality of the classical potential flow formulation of the water wave problem, (2.1), by considering surface quantities as unknowns. We begin with the observation that when the free surface $\eta(x, t)$, Dirichlet data at the free surface $\xi(x, t)=\varphi(x, \eta(x, t), t)$, and Neumann data at the bottom $v(x, t)$ are specified, we can in principle solve the full problem, since $\varphi$ satisfies Laplace's equation with appropriate boundary conditions [32]. In this way, the water wave problem can be reduced from one posed inside the entire fluid domain to one posed at the free surface alone. This fact was originally noted by Zakharov [50] (for deep water), who reformulated (2.1) as a Hamiltonian system in terms of the canonically conjugate variables $\eta$ and $\xi$.

This surface formulation plays a central role in the efficiency of the numerical method developed here to solve (2.1). For expository convenience, we will first describe the case of a fixed (static) bottom, based on the previous work of Craig et al. [10] and Guyenne and Nicholls [28]. Our new formulation of the more general case of a moving (time-dependent) bottom will be addressed separately in a subsequent section.

2.1. Static bottom topography. To begin the description of our boundary formulation, we define the DNO, which, for the solution of Laplace's equation (2.1a), maps Dirichlet data to Neumann data at the free surface, by

$$
G(\beta, \eta) \xi=\left.\nabla \varphi\right|_{y=\eta} \cdot \nu(\eta)
$$

where $\nu(\eta)=\left(-\nabla_{x} \eta, 1\right)$ is a (nonnormalized) exterior vector normal to the free surface. This operator is linear in $\xi$, but it is nonlinear with explicit nonlocal dependence on $\beta$ and $\eta$, which determine the fluid domain. Expressing the velocity potential and its derivatives on the free surface in terms of $\xi$ and $G(\beta, \eta) \xi$, the following set of equations equivalent to (2.1) is obtained (see [13]):

$$
\begin{aligned}
\partial_{t} \eta= & G(\beta, \eta) \xi \\
\partial_{t} \xi= & -g \eta-\frac{1}{2\left(1+\left|\nabla_{x} \eta\right|^{2}\right)}\left[\left|\nabla_{x} \xi\right|^{2}-(G(\beta, \eta) \xi)^{2}\right. \\
& \left.-2(G(\beta, \eta) \xi) \nabla_{x} \xi \cdot \nabla_{x} \eta+\left|\nabla_{x} \xi\right|^{2}\left|\nabla_{x} \eta\right|^{2}-\left(\nabla_{x} \xi \cdot \nabla_{x} \eta\right)^{2}\right] .
\end{aligned}
$$

This is a Hamiltonian system in Zakharov's formulation of the water wave problem [50], with Hamiltonian

$$
\mathcal{H}=\frac{1}{2} \int_{\mathbb{R}}\left[\xi G(\beta, \eta) \xi+g \eta^{2}\right] d x .
$$

The analyticity of the DNO has been studied by many authors, beginning with the work of Coifman and Meyer [8], which built on the results of Calderón [5]. For $\beta=0$, Coifman and Meyer showed that if $\eta \in \operatorname{Lip}(\mathbb{R})$, then $G$ is an analytic function of $\eta$, from which it follows that $G$ can be written in terms of a convergent Taylor series

$$
G(\eta)=\sum_{l=0}^{\infty} G^{(l)}(\eta)
$$


for $\eta$ sufficiently small, where each term $G^{(l)}$ is homogeneous of degree $l$. Craig, Schanz, and Sulem [12] extended these results for periodic $\eta \in C^{1}\left(\mathbb{R}^{2}\right)$, and Craig and Nicholls [11] generalized this argument for $\eta \in C^{1}\left(\mathbb{R}^{n-1}\right)$. Nicholls and Reitich [42] devised a direct method to estimate the $G^{(l)}$ (requiring the slightly stronger hypothesis $\left.\eta \in C^{3 / 2+\delta}\left(\mathbb{R}^{n-1}\right)\right)$ with the goal of stabilized high-order calculations. These highorder results were exhibited in [43], and in [44] joint spatial and parametric analyticity results were established. The most comprehensive results to date have been derived by Hu and Nicholls [29], who used a finer analysis of the Nicholls-Reitich recursions to permit data in Hölder classes and a Lipschitz profile. A recursion formula for the lth-order term in (2.4) is given in Craig and Sulem [13] in two dimensions, and the straightforward generalization to three dimensions was derived by Nicholls [41].

For $l$ odd,

$$
\begin{aligned}
G^{(l)}= & |D|^{l-1} D \frac{\eta^{l}}{l !} \cdot D-\sum_{j=2, \text { even }}^{l-1}|D|^{j} \frac{\eta^{j}}{j !} G^{(l-j)} \\
& -\sum_{j=1, \text { odd }}^{l}|D|^{j-1} G^{(0)} \frac{\eta^{j}}{j !} G^{(l-j)}
\end{aligned}
$$

and, for $l>0$ even,

$$
\begin{aligned}
G^{(l)}= & |D|^{l-2} G^{(0)} D \frac{\eta^{l}}{l !} \cdot D-\sum_{j=2, \text { even }}^{l}|D|^{j} \frac{\eta^{j}}{j !} G^{(l-j)} \\
& -\sum_{j=1, \text { odd }}^{l-1}|D|^{j-1} G^{(0)} \frac{\eta^{j}}{j !} G^{(l-j)},
\end{aligned}
$$

where $G^{(0)}=|D| \tanh \left(h_{0}|D|\right)$ and $D=-i \nabla_{x}$.

In the case of nontrivial bottom topography $(\beta \neq 0)$ the results listed above have recently been extended by Nicholls and Taber [45], who showed that the DNO in such a configuration is jointly analytic in all spatial and parametric (boundary) variables and that it can be analytically continued in the two parametric variables. The expansions listed above, (2.5), have recently been extended to the case of an uneven bottom by Craig et al. [10], who showed that they can be used verbatim with the exception that the first term $G^{(0)}$ is replaced by

$$
G^{(0)}=|D| \tanh \left(h_{0}|D|\right)+|D| L(\beta) .
$$

The operator $|D| L(\beta)$, which takes into account the bottom variation, is derived from the Neumann boundary condition at the bottom (2.1d) for $v=0$. By an extension of the argument in [42], it follows that $|D| L$ can also be expressed as a convergent Taylor series expansion in $\beta$,

$$
|D| L(\beta)=\sum_{j=0}^{\infty}|D| L_{j}(\beta),
$$

where each term $|D| L_{j}$ can be determined explicitly by a recursion formula as given in [10]. 
We propose here an alternate recursive formulation of $|D| L$ which proves much more suitable for numerical simulations. Using the transformation

$$
|D| L_{j}=\operatorname{sech}\left(h_{0}|D|\right)|D| F_{j}
$$

and rearranging terms in the recursion formula of $|D| L_{j}$ (see [10]), we obtain the following: for $j$ odd,

$$
\begin{aligned}
|D| F_{j}=-D \cdot[ & \frac{\beta^{j}}{j !} \operatorname{sech}\left(h_{0}|D|\right)|D|^{j-1} D+\sum_{l=2, \text { even }}^{j-1} \frac{\beta^{l}}{l !}|D|^{l-2} D\left(|D| F_{j-l}\right) \\
& \left.-\sum_{l=1, \text { odd }}^{j-2} \frac{\beta^{l}}{l !} \tanh \left(h_{0}|D|\right)|D|^{l-2} D\left(|D| F_{j-l}\right)\right]
\end{aligned}
$$

and, for $j>0$ even,

$$
\begin{aligned}
|D| F_{j}=-D \cdot & {\left[\sum_{l=2, \text { even }}^{j-2} \frac{\beta^{l}}{l !}|D|^{l-2} D\left(|D| F_{j-l}\right)\right.} \\
& \left.-\sum_{l=1, \text { odd }}^{j-1} \frac{\beta^{l}}{l !} \tanh \left(h_{0}|D|\right)|D|^{l-2} D\left(|D| F_{j-l}\right)\right] .
\end{aligned}
$$

The motivation for introducing $|D| F_{j}$ is that the new recursion formulae (2.7) involve bounded and smoothing operators (i.e., $\tanh \left(h_{0}|D|\right)$ and $\operatorname{sech}\left(h_{0}|D|\right)$ ), while the corresponding formulae for $|D| L_{j}$ in [10] exhibit "exponentially unbounded" operators (i.e., $\cosh \left(h_{0}|D|\right)$ and $\left.\sinh \left(h_{0}|D|\right)\right)$ which are not well defined for any function of finite smoothness. For example, if $f \in H^{s}$ for any $s<\infty$, then $\cosh \left(h_{0}|D|\right) f \notin L^{2}$. As a consequence, the evaluation of $|D| L_{j}$ through $|D| F_{j}$ is numerically more robust and stable, especially with the use of a pseudospectral method as described in section 3 .

2.2. Moving bottom topography. We now turn our attention to the more general case where the bottom perturbation $\beta$ depends on both space and time (which was not treated in $[10,28]$ ). The solution of Laplace's equation is now determined by Dirichlet data $\xi$ at the free surface and Neumann data (2.1d) at the bottom. Accordingly, we note that the DNO now depends linearly upon two quantities, the Dirichlet data $\xi$ and the bottom velocity $v$,

$$
G(\beta, \eta)[\xi, v]=\left.\nabla \varphi\right|_{y=\eta} \cdot \nu(\eta)
$$

which maps $(\xi, v)$ to Neumann data at the free surface.

Using an argument almost identical to the one used by Nicholls and Taber [45], we can establish that this new DNO depends analytically jointly upon the (time-frozen) functions $\beta$ and $\eta$, provided that the (time-frozen) data $\xi$ and $v$ are sufficiently regular. To be more precise, if we consider $\beta=\delta b$ and $\eta=\varepsilon f$ to be small perturbations, we can expand the DNO in the Taylor series

$$
G(\beta, \eta)[\xi, v]=G(\delta b, \varepsilon f)[\xi, v]=\sum_{m_{0}, m_{1}=0}^{\infty} G^{\left(m_{0}, m_{1}\right)}(b, f)[\xi, v] \delta^{m_{0}} \varepsilon^{m_{1}},
$$

which converges strongly in the sense of the following theorem.

Copyright $@$ by SIAM. Unauthorized reproduction of this article is prohibited. 
TheOREM 2.1. Given any integer $s \geq 0$, if $b, f \in C^{s+2}, \xi \in H^{s+3 / 2}$, and $v \in H^{s+1 / 2}$, then $G^{\left(m_{0}, m_{1}\right)}(b, f)[\xi, v] \in H^{s+1 / 2}$ and

$$
\left\|G^{\left(m_{0}, m_{1}\right)}(b, f)[\xi, v]\right\|_{H^{s+1 / 2}} \leq K B_{0}^{m_{0}} B_{1}^{m_{1}}
$$

for constants $K, B_{0}, B_{1}>0$.

Proof. As we mentioned above, the proof is nearly identical to the one presented in [45]. Considering the elliptic boundary value problem

$$
\begin{aligned}
\Delta w=0 \quad & \text { in } S(\delta b, \varepsilon f), \\
w(x, \varepsilon f)=\xi(x) & \text { at } y=\varepsilon f, \\
\nabla w \cdot \nu(\delta b)=v(x) & \text { at } y=-h_{0}+\delta b,
\end{aligned}
$$

which defines the DNO,

$$
G(\delta b, \varepsilon f)[\xi, v]=\left.\nabla w\right|_{y=\varepsilon f} \cdot \nu(\varepsilon f),
$$

we begin with a "domain flattening" transformation

$$
x^{\prime}=x, \quad y^{\prime}=h_{0}\left(\frac{y-\varepsilon f}{h_{0}-\delta b+\varepsilon f}\right) .
$$

This produces a transformed field

$$
u\left(x^{\prime}, y^{\prime} ; \delta, \varepsilon\right)=w\left(x^{\prime},\left(h_{0}-\delta b\left(x^{\prime}\right)+\varepsilon f\left(x^{\prime}\right)\right) y^{\prime} / h_{0}+\varepsilon f\left(x^{\prime}\right)\right),
$$

which can be expanded in a double Taylor series

$$
u\left(x^{\prime}, y^{\prime} ; \delta, \varepsilon\right)=\sum_{m_{0}, m_{1}=0}^{\infty} u^{\left(m_{0}, m_{1}\right)}\left(x^{\prime}, y^{\prime}\right) \delta^{m_{0}} \varepsilon^{m_{1}}
$$

Equation (2.10) in the transformed coordinates produces recursions for the $u^{\left(m_{0}, m_{1}\right)}$ which differ from those found in [45] only in that the velocity function $v$ appears on the right-hand side. A straightforward modification of the elliptic estimate found in [45] enables the inductive approach outlined in the rest of that paper, and the analyticity result follows quickly, provided that $v$ sits in the proper function space, $H^{s+1 / 2}$.

One method for numerically simulating the action of the DNO is to derive forms for the $G^{\left(m_{0}, m_{1}\right)}$ in (2.9) which can be conveniently incorporated into a numerical algorithm. For the spectral collocation approach we advocate in this paper, several design philosophies can be used to find these formulae (e.g., operator expansions, field expansions, transformed field expansions; see [42, 43, 44, 9]). Here we follow the operator expansions method devised by Milder [38] and Craig and Sulem [13]; we refer the interested reader to the work of Nicholls and Reitich [42, 43, 44] for a detailed description of each approach together with an assessment of its strengths and weaknesses.

To make the presentation more clear we point out that the DNO can be written as the sum of two terms, one $\left(G_{1}\right)$ which is linear in $\xi$ and independent of $v$ and the second $\left(G_{2}\right)$ which is linear in $v$ and independent of $\xi$; both are, of course, nonlinearly dependent on both $\beta$ and $\eta$. One way to recognize this decomposition is to examine 
(2.10) and notice that one can write $w=w_{1}+w_{2}$, where $w_{1}$ satisfies (2.10) with $v$ identically zero, and $w_{2}$ satisfies (2.10) with $\xi$ identically zero.

To find formulae for the $G_{1}^{\left(m_{0}, m_{1}\right)}$ and $G_{2}^{\left(m_{0}, m_{1}\right)}$, consider the family of harmonic functions

$$
\varphi_{k}(x, y)=\left(a_{k} e^{|k| y}+b_{k} e^{-|k| y}\right) e^{i k \cdot x},
$$

which satisfy Laplace's equation. To find $G_{1}$ and $G_{2}$ it is most convenient to make two different choices for the constants $\left(a_{k}, b_{k}\right)$; first, for $G_{1}$, we set

$$
\left(a_{k}, b_{k}\right)=\left(\frac{e^{|k| h}}{e^{-|k| h}+e^{|k| h}}, \frac{e^{-|k| h}}{e^{-|k| h}+e^{|k| h}}\right)
$$

so that $\varphi_{k}=e^{i k \cdot x}$ at $y=0$ and $\partial_{y} \varphi_{k}=0$ at $y=-h_{0}$. For $G_{2}$, we set

$$
\left(a_{k}, b_{k}\right)=\left(\frac{1}{|k|\left(e^{-|k| h}+e^{|k| h}\right)}, \frac{-1}{|k|\left(e^{-|k| h}+e^{|k| h}\right)}\right)
$$

so that $\varphi_{k}=0$ at $y=0$ and $\partial_{y} \varphi_{k}=e^{i k \cdot x}$ at $y=-h_{0}$. Now we insert (2.9), (2.11), and (2.12) into (2.8) together with the boundary values

$$
\xi(x)=\varphi_{k}(x, \eta(x)), \quad v(x)=\left.\nabla \varphi_{k}\right|_{y=-h} \cdot \nu(\beta) .
$$

Expanding all terms about $\beta, \eta=0$ and identifying contributions of the same degree in $\beta$ and $\eta$, this yields, at zeroth order $\left(m_{0}=m_{1}=0\right)$,

$$
G_{1}^{(0,0)}=|D| \tanh \left(h_{0}|D|\right), \quad G_{2}^{(0,0)}=\operatorname{sech}\left(h_{0}|D|\right),
$$

where we have used the equivalence $k \leftrightharpoons D[13,42,9,10]$. As expected, $G_{1}^{(0,0)}$ is identical to $G^{(0)}$ for $\eta=0$. There is a recursion formula for the higher-order terms in the Taylor series expansions of $G_{1}$ and $G_{2}$ (provided below), and we distinguish two cases. The first is the special case where either $m_{0}=0$ or $m_{1}=0$, and the second is the more general case where neither $m_{0}=0$ nor $m_{1}=0$. The expressions are

(i) $m_{0} \neq 0, m_{1}=0$ :

$$
G_{1}^{\left(m_{0}, 0\right)}=-\sum_{l=1, \text { odd }}^{m_{0}} \operatorname{sech}\left(h_{0}|D|\right)|D|^{l-1} D \frac{\beta^{l}}{l !} \cdot D G_{2}^{\left(m_{0}-l, 0\right)}
$$

and

$$
\begin{aligned}
G_{2}^{\left(m_{0}, 0\right)}= & -\sum_{l=2, \text { even }}^{m_{0}}|D|^{l-2} D \frac{\beta^{l}}{l !} \cdot D G_{2}^{\left(m_{0}-l, 0\right)} \\
& +\sum_{l=1, \text { odd }}^{m_{0}}|D|^{l-2} \tanh \left(h_{0}|D|\right) D \frac{\beta^{l}}{l !} \cdot D G_{2}^{\left(m_{0}-l, 0\right)} .
\end{aligned}
$$

(ii) $m_{0}=0, m_{1} \neq 0: m_{1}$ odd,

$$
\begin{aligned}
G_{1}^{\left(0, m_{1}\right)}= & |D|^{m_{1}-1} D \frac{\eta^{m_{1}}}{m_{1} !} \cdot D-\sum_{l=2, \text { even }}^{m_{1}-1}|D|^{l} \frac{\eta^{l}}{l !} G_{1}^{\left(0, m_{1}-l\right)} \\
& -\sum_{l=1, \text { odd }}^{m_{1}}|D|^{l} \tanh \left(h_{0}|D|\right) \frac{\eta^{l}}{l !} G_{1}^{\left(0, m_{1}-l\right)}
\end{aligned}
$$

Copyright $@$ by SIAM. Unauthorized reproduction of this article is prohibited. 
and

$$
G_{2}^{\left(0, m_{1}\right)}=-\sum_{l=1, \text { odd }}^{m_{1}} \operatorname{sech}\left(h_{0}|D|\right)|D|^{l-1} \frac{\eta^{l}}{l !} G_{1}^{\left(0, m_{1}-l\right)}
$$

(iii) $m_{0}=0, m_{1} \neq 0: m_{1}>0$ even,

$$
\begin{aligned}
G_{1}^{\left(0, m_{1}\right)}= & |D|^{m_{1}-1} \tanh \left(h_{0}|D|\right) D \frac{\eta^{m_{1}}}{m_{1} !} \cdot D \\
& -\sum_{l=2, \text { even }}^{m_{1}}|D|^{l} \frac{\eta^{l}}{l !} G_{1}^{\left(0, m_{1}-l\right)} \\
& -\sum_{l=1, \text { odd }}^{m_{1}-1}|D|^{l} \tanh \left(h_{0}|D|\right) \frac{\eta^{l}}{l !} G_{1}^{\left(0, m_{1}-l\right)}
\end{aligned}
$$

and

$$
\begin{aligned}
G_{2}^{\left(0, m_{1}\right)}= & \operatorname{sech}\left(h_{0}|D|\right)|D|^{m_{1}-2} D \frac{\eta^{m_{1}}}{m_{1} !} \cdot D \\
& -\sum_{l=1, \text { odd }}^{m_{1}-1} \operatorname{sech}\left(h_{0}|D|\right)|D|^{l-1} \frac{\eta^{l}}{l !} G_{1}^{\left(0, m_{1}-l\right)}
\end{aligned}
$$

(iv) $m_{0}, m_{1} \neq 0$ :

$$
\begin{aligned}
G_{1}^{\left(m_{0}, m_{1}\right)}= & -\sum_{l=2, \text { even }}^{m_{1}}|D|^{l} \frac{\eta^{l}}{l !} G_{1}^{\left(m_{0}, m_{1}-l\right)} \\
& -\sum_{l=1, \text { odd }}^{m_{1}}|D|^{l} \tanh \left(h_{0}|D|\right) \frac{\eta^{l}}{l !} G_{1}^{\left(m_{0}, m_{1}-l\right)} \\
& -\sum_{l=1, \text { odd }}^{m_{0}} \operatorname{sech}\left(h_{0}|D|\right)|D|^{l-1} D \frac{\beta^{l}}{l !} \cdot D G_{2}^{\left(m_{0}-l, m_{1}\right)}
\end{aligned}
$$

and

$$
\begin{aligned}
G_{2}^{\left(m_{0}, m_{1}\right)}= & -\sum_{l=1, \text { odd }}^{m_{1}} \operatorname{sech}\left(h_{0}|D|\right)|D|^{l-1} \frac{\eta^{l}}{l !} G_{1}^{\left(m_{0}, m_{1}-l\right)} \\
& -\sum_{l=2, \text { even }}^{m_{0}}|D|^{l-2} D \frac{\beta^{l}}{l !} \cdot D G_{2}^{\left(m_{0}-l, m_{1}\right)} \\
& +\sum_{l=1, \text { odd }}^{m_{0}}|D|^{l-2} \tanh \left(h_{0}|D|\right) D \frac{\beta^{l}}{l !} \cdot D G_{2}^{\left(m_{0}-l, m_{1}\right)}
\end{aligned}
$$

Copyright (C) by SIAM. Unauthorized reproduction of this article is prohibited. 
We note again the presence of the operator $\operatorname{sech}\left(h_{0}|D|\right)$ in the above equations, which has a stabilizing effect on the calculation of the DNO. It can be checked that recursion formulae (2.14c) and (2.14e) for $G_{1}$ correspond to (2.5) for the DNO in the case of a static, uniform bottom $(\beta=0, v=0)$. Moreover, expressions (2.14a) $-(2.14 \mathrm{~b})$ are equivalent to $(2.6)$ for $G^{(0)}$ (with $|D| L$ given by $(2.7)$ through $|D| F_{j}$ ) in the case of an undisturbed free surface over static bottom topography $(\eta=0, v=0)$.

Remark. The equations of motion equivalent to (2.2) for moving bottom topography are obtained by simply replacing $G(\beta, \eta) \xi$ by $G(\beta, \eta)[\xi, v]$ in $(2.2)$. The analogue of the Hamiltonian (2.3) is given by

$$
\mathcal{H}=\frac{1}{2} \int_{\mathbb{R}}\left[\xi G(\beta, \eta)[\xi, v]-\zeta v+g \eta^{2}\right] d x,
$$

where $\zeta$ and $v$ are the Dirichlet and Neumann data at the bottom, respectively. The negative sign for the second term in (2.15) is due to the fact that $v$ is defined as the upward (interior) normal velocity at the bottom (see (2.1d)). The inclusion of $\zeta$ in (2.15) requires the existence of a "Neumann-Dirichlet operator" which, given $\beta, \eta, \xi$, and $v$ (Neumann data), produces the Dirichlet data $\zeta$. It is not difficult to show that such an operator does exist and is well defined under reasonable hypotheses.

3. Numerical methods. In this section, we describe the numerical methods for space and time discretizations of the model. We also discuss the implementation of techniques for wave generation and wave absorption to deal with nonperiodic problems.

3.1. Spatial discretization. We begin by considering the classical periodic boundary conditions in the $x$-direction and use a pseudospectral method for spatial discretization of the problem $[23,6,13,41]$. This is a natural choice for the computation of the DNO, since each term in its Taylor series expansions consists of concatenations of Fourier multipliers with powers of $\beta$ and $\eta$. For static bottom topography, both operators $G$ and $|D| L$ are approximated by a finite number of terms in their Taylor series,

$$
G(\beta, \eta) \simeq \sum_{m_{1}=0}^{M} G^{\left(m_{1}\right)}(\beta, \eta), \quad|D| L(\beta) \simeq \sum_{m_{0}=0}^{M_{b}}|D| L_{m_{0}}(\beta),
$$

which is equivalent to

$$
G(\beta, \eta) \simeq G^{M_{b}, M}(\beta, \eta) \equiv \sum_{m_{0}=0}^{M_{b}} \sum_{m_{1}=0}^{M} G^{\left(m_{0}, m_{1}\right)}(\beta, \eta),
$$

where $M$ and $M_{b}$ are independently chosen according to the physical problem under consideration. The same procedure applies to the approximation of (2.9) for moving bottom topography, with $0 \leq m_{0} \leq M_{b}$ and $0 \leq m_{1} \leq M$. The joint analyticity properties of the DNO (Theorem 2.1) imply

$$
\begin{aligned}
\left\|G-G^{M_{b}, M}\right\|_{H^{s+1 / 2}} & \leq \sum_{\left\{m_{0}>M_{b}\right\} \text { or }\left\{m_{1}>M\right\}}\left\|G^{\left(m_{0}, m_{1}\right)}\right\|_{H^{s+1 / 2}} \\
& \leq B_{0}^{M_{b}} B_{1}^{M} \sum_{\left\{m_{0}>M_{b}\right\} \text { or }\left\{m_{1}>M\right\}} K B_{0}^{m_{0}-M_{b}} B_{1}^{m_{1}-M} \\
& \leq \tilde{K} B_{0}^{M_{b}} B_{1}^{M},
\end{aligned}
$$

Copyright $\odot$ by SIAM. Unauthorized reproduction of this article is prohibited. 
i.e., the exponential convergence of the series expansions. The constants $K, B_{0}$, and $B_{1}$ (and thus the rate of the convergence of our approximation) depend primarily upon the size and smoothness of the bottom and top deformations, $\beta$ and $\eta$, respectively. Consequently, for large or rough topography and/or surface waves one must incorporate more terms. In the case of static topography we have never found this to be a particularly steep cost for our algorithm; however, for moving bottoms it can be more severe (see section 3.2). However, as the rate of convergence is quite rapid (exponential) we have typically found it sufficient to select a small number of perturbation terms, $M_{b}$ and $M$, for quite accurate results.

To compute these Taylor terms, the three quantities $\eta, \xi$, and $\beta$ are approximated by truncating their Fourier series with the same number of modes, $N$. Applications of Fourier multipliers are performed in Fourier space, while nonlinear products are calculated in physical space at a discrete set of equally spaced points. For example, application of the zeroth-order operators $G_{1}^{(0,0)}$ and $G_{2}^{(0,0)}$ in (2.13) is equivalent to multiplication by $|k| \tanh \left(h_{0}|k|\right)$ and $\operatorname{sech}\left(h_{0}|k|\right)$ in Fourier space, respectively. A zeropadding technique is used to prevent aliasing errors in the calculation of the nonlinear terms [6], and all operations are performed using the FFTW library [21].

As pointed out in [13], expansions of the DNO as performed here are uniform in wavenumber. Thus there are no a priori restrictions on the relative length scales between bottom and surface variations. In particular, the method is not restricted to slowly varying topography compared to surface waves. However, because of the character of the Taylor series expansions and the use of fast Fourier transforms, bottom and surface variations are both required to be single-valued (e.g., no overturning waves), sufficiently smooth, and of moderate amplitude.

3.2. Time integration. Time integration is carried out in Fourier space. The linear terms in (2.2) are solved exactly by an integrating factor technique [13, 1]. The nonlinear terms are integrated in time using a fourth-order Runge-Kutta scheme with constant step size.

For more efficiency in the case of static bottom topography, the operator $|D| L$ can be precalculated and saved before time integration begins. It can then be used (at no extra cost) in the calculation of the DNO at every time step. The computational cost is therefore essentially determined by the evaluation of (2.4) and (2.5). For perturbations up to order $M$, the number of operations required is $O\left(M^{2} N \ln N\right)$ per time step. In the case of moving bottom topography, the computational cost is higher and the number of operations required can be estimated from (2.9) and (2.14) to be $O\left(\max \left(M, M_{b}\right) M M_{b} N \ln N\right)$ per time step.

In the computations (especially of large-amplitude waves; see section 4.2), it was observed that spurious oscillations can develop in the wave profile, due to the onset of an instability related to the growth of numerical errors at high wavenumbers. This may be attributed to some ill-conditioning of the operator expansion method for the DNO $[42,43,44]$. Similar high-wavenumber instabilities were observed by other authors (e.g., $[19,1]$ ), who used smoothing techniques to circumvent this difficulty. Here, at every time step, we apply an ideal low-pass filter to $\hat{\eta}_{k}$ and $\hat{\xi}_{k}$ of the form

$$
\gamma_{k}=\left\{\begin{array}{lll}
1 & \text { if }|k| / k_{\max } \leq \theta, \quad 0<\theta \leq 1, \\
0 & \text { if }|k| / k_{\max }>\theta,
\end{array}\right.
$$

where $k_{\max }$ is the largest wavenumber of the spectrum. Typically, we found that $\theta=0.8$ suffices to stabilize the solution. Care was taken to specify a sufficiently high 
spatial resolution and a value of $\theta$ close to unity so that only energy levels located in the high-wavenumber region of the spectrum are suppressed by filtering. This ideal low-pass filter also further contributes to removal of aliasing errors. We point out that, in most of our simulations with small to moderate amplitudes (e.g., with incident Stokes waves as in sections 4.3 and 4.4), no filtering was used at all.

3.3. Lateral boundary conditions. In the present scheme, adopting a pseudospectral method allows for an efficient evaluation of the DNO and equations of motion via the fast Fourier transform, but this requires periodic boundary conditions in the $x$-direction. A simple way of overcoming this limitation is to increase the size of the computational domain until the effect of the boundaries becomes negligible. This method, however, has a limited range of applicability and is not suitable for longtime simulations. Here we propose efficient methods for wave generation and wave absorption in a periodic domain using relaxation zones [4]. The goal is to extend the range of applicability of the algorithm to more realistic situations; for example, these methods are used to generate and dissipate Stokes waves in a channel for comparison with laboratory experiments in sections 4.3 and 4.4.

The principle of relaxation zones is to relax the numerical solution towards a specified analytical solution over a limited region near the boundary. This is achieved by simply defining a relaxation coefficient $0 \leq c_{r}(x) \leq 1$ and an exact desired solution $(\widetilde{\eta}, \widetilde{\xi})$. At each time step, the solution within the relaxation zone is then redefined to be

$$
\left(\begin{array}{l}
\eta(x, t) \\
\xi(x, t)
\end{array}\right)=c_{r}(x)\left(\begin{array}{c}
\eta(x, t) \\
\xi(x, t)
\end{array}\right)+\left[1-c_{r}(x)\right]\left(\begin{array}{c}
\widetilde{\eta}(x, t) \\
\widetilde{\xi}(x, t)
\end{array}\right) .
$$

In the two-dimensional applications presented here, the computational domain is specified in the following way: an absorbing zone at both ends of the domain and a wavemaker zone near the left end, adjacent to the first absorbing zone. A typical relaxation coefficient we used is given by

$$
c_{r}(x)=\frac{1}{2}+\frac{1}{2} \tanh \left(2 \pi\left(\frac{x}{L}-\frac{1}{2}\right)\right),
$$

which is defined in a region of characteristic length $L$, which is chosen long enough to span at least 3-4 wavelengths. For wave generation, the specified solution is the thirdorder Stokes wave and, for wave absorption, $(\widetilde{\eta}, \widetilde{\xi})=(0,0)$. This technique is easy to implement and is very effective, as illustrated in Figure 3.1. An incident Stokes wave

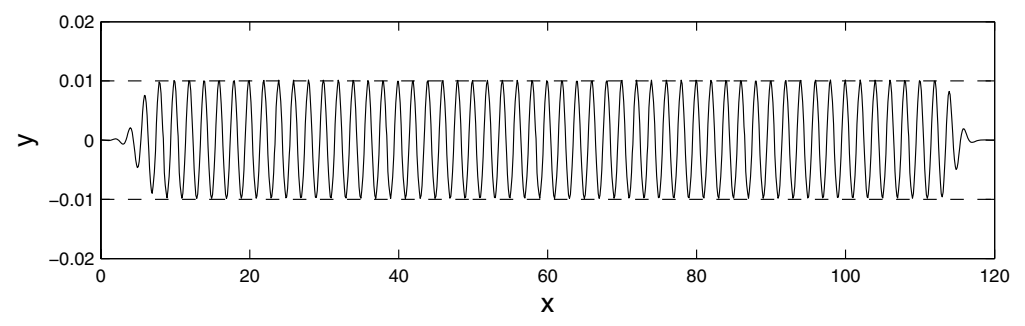

FIG. 3.1. Wave generation and absorption using relaxation zones. The incident wave is a Stokes wave of amplitude $a_{0} / h_{0}=0.01$ and wavenumber $k_{0} h_{0}=\pi$, moving from left to right on constant depth. The solution at $t / \sqrt{h_{0} / g}=600$ is a near-perfect, steadily progressing wave. 


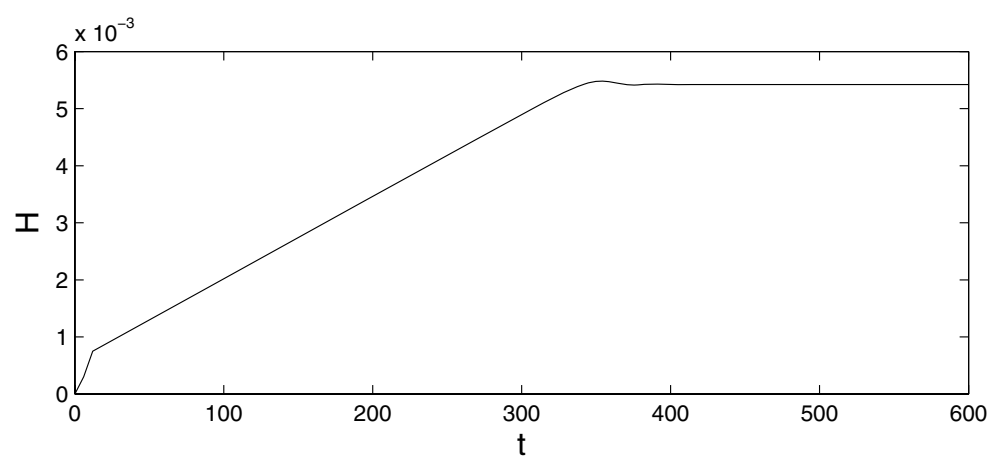

FIG. 3.2. Time evolution of the Hamiltonian, (2.3), for a Stokes wave of amplitude $a_{0} / h_{0}=0.01$ and wavenumber $k_{0} h_{0}=\pi$ generated and absorbed using relaxation zones. A steady state is reached around $t / \sqrt{h_{0} / g}=400$.

of amplitude $a_{0} / h_{0}=0.01$ and wavenumber $k_{0} h_{0}=\pi$ is specified, moving from left to right on constant depth $\left(M=4, M_{b}=0, L=10\right)$. The result at $t / \sqrt{h_{0} / g}=600$ is a near-perfect, steadily progressing wave between the two absorbing zones at the extremities of the domain. It is shown in Figure 3.2 that the solution has reached a steady state and the Hamiltonian (2.3) is well conserved. This clearly indicates that the outgoing wave is absorbed well by the relaxation zones and negligible wave reflection is produced.

Another effective method for wave absorption which can be easily incorporated into the present algorithm is the so-called sponge layer or numerical beach $[46,7]$. This consists in (explicitly) adding localized damping terms (e.g., pressure terms) in the equations of motion (2.2). We have performed computations with a damping term of the form

$$
-\left.\frac{1}{\sqrt{1+\left|\nabla_{x} \eta\right|^{2}}} \sqrt{g h_{0}}\left(\frac{x}{L}\right)^{2} \nabla \varphi\right|_{y=\eta} \cdot \nu(\eta)
$$

added to the right-hand side of (2.2b), which also gives good results (not shown here).

Note that we have not attempted to model in detail wavemaker and absorbing boundary conditions as used in laboratory experiments, and it would be worthwhile to consider more sophisticated methods for wave generation and absorption (or radiation) in the future. The primary goal in this paper is rather to show that our numerical method is flexible and robust and can be adapted to a variety of water wave problems with bottom topography. The following applications demonstrate the practical effectiveness of our methods.

4. Numerical results. In this section, we present a series of two-dimensional $(n=2)$ simulations to demonstrate the performance of our numerical method. The first four examples consider the case of static bottom topography, while the last deals with moving bathymetry. The accuracy of the computations are assessed by comparison with analytical and experimental results. Unless stated otherwise, all variables are nondimensionalized according to long-wave theory; i.e., lengths are divided by $h_{0}$ and times divided by $\sqrt{h_{0} / g}$.

4.1. Linear wave shoaling. We begin by studying the linear wave shoaling problem in which linear waves are distorted when propagating from deep to shallow 
(a)
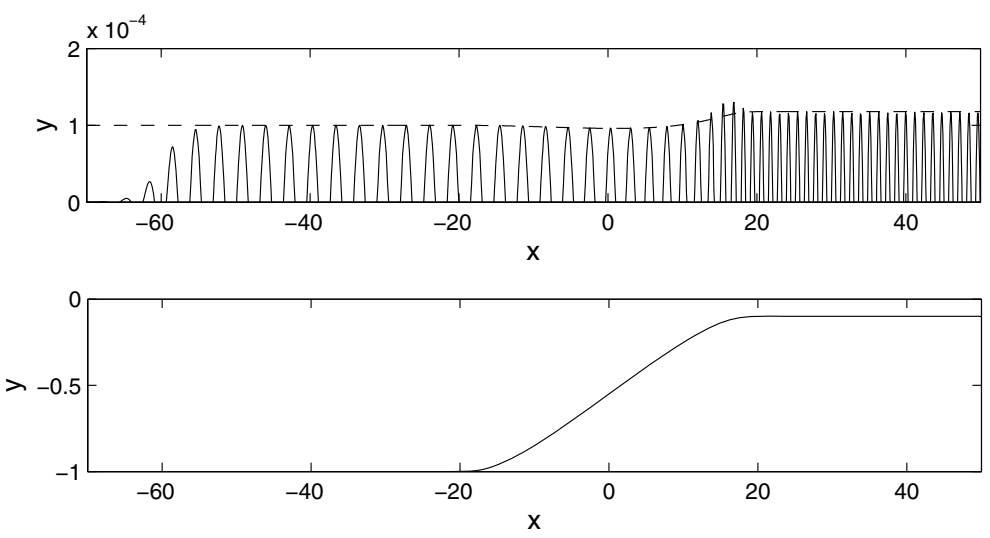

(b)

FIG. 4.1. Shoaling of a linear wave of amplitude $a_{0}=10^{-4}$ and wavenumber $k_{0}=2$ over a smooth bathymetry: (a) comparison between the computed wave profile (solid line) and the envelope predicted by linear theory (dashed line) at $t=500$ in the steady state; (b) bottom profile.

water. We specify a smooth bathymetry given by

$$
\beta(x)=\frac{h_{0}-h_{1}}{2}\left[1+\tanh \left(\frac{\sin (\pi x / L)}{1-(2 x / L)^{2}}\right)\right],
$$

as shown in Figure 4.1(b), with $h_{0}=1, h_{1}=0.1$, and $L=45$. A linear wave of amplitude $a_{0}=10^{-4}$ and wavenumber $k_{0}=2$ is generated at the left end of the domain and absorbed at the opposite end to prevent reflection. A bottom topography of large amplitude is generally specified in such an experiment in order to describe the transition from deep to shallow water. Accordingly, we choose a large value of $M_{b}$, so that the linear waves "feel" the effects of the bottom variations.

Figure 4.1(a) shows the computed wave profile in the steady state $\left(M=0, M_{b}=\right.$ $15)$, along with the amplitude envelope predicted by energy conservation $[16,33]$ :

$$
\frac{a}{a_{0}}=\left[\frac{k\left(1+2 h_{0} k_{0} / \sinh \left(2 h_{0} k_{0}\right)\right)}{k_{0}(1+2 h k / \sinh (2 h k))}\right]^{1 / 2} .
$$

We see that the wave amplitude first decreases slightly over the bottom slope and then rapidly increases and stabilizes over the plateau at a level higher than the incident one. Such features agree very well with the linear prediction (4.1). That the wavenumber increases as the wave travels from deep to shallow water is also an expected phenomenon [4, 34]. A few wave crests slightly exceeding the predicted amplitude and occurring over the top of the slope may be explained by the (small) amplification of numerical harmonics during the shoaling process.

4.2. Solitary wave shoaling on a slope. Our second experiment concerns the evolution of solitary waves traveling up a plane slope, and our computations are compared with results obtained by a boundary element (BE) method [25, 24, 27]. The latter method has been extensively tested and validated against both theoretical and experimental results. The bottom geometry is specified as follows: a uniform depth $h_{0}$ for $x<d$, and a slope $s$ for $x \geq d$ (Figure 4.2); the initial condition is a fully nonlinear solitary wave of height $a_{0}$ computed by Tanaka's method [47]. This corresponds to a numerically exact and permanent solution of nonlinear potential flow theory, and we have checked that such a wave propagates with negligible change of 


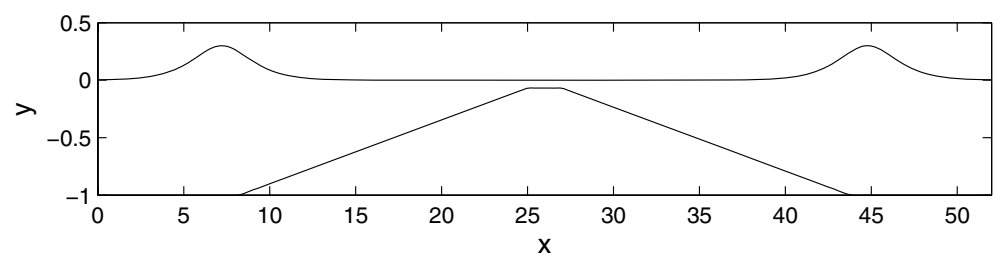

FIG. 4.2. Bottom topography and initial condition in the solitary wave shoaling problem. The two solitary waves are of amplitude $a_{0}=0.3$ and the bottom slope is $s=1 / 18$.

(a)
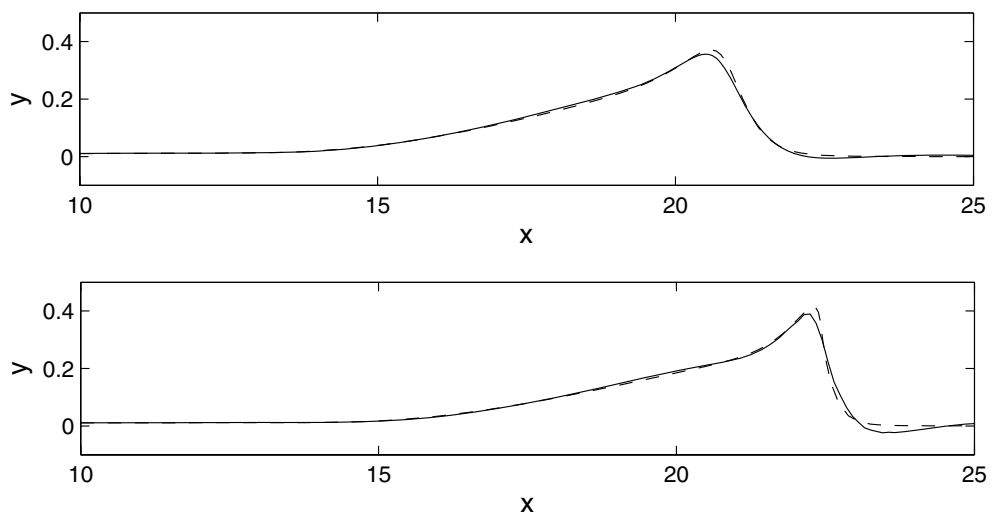

(b)

FIG. 4.3. Comparison of solitary wave profiles between boundary element (dashed line) and spectral (solid line) methods for $a_{0}=0.3$ at times (a) $t=12$ and (b) $t=13.6$.

shape and speed on uniform depth in our model. Because rigid lateral boundaries are used in the BE code, both the bottom geometry and the initial condition in the spectral model are specified symmetrically about the center of the domain in order to simulate a rigid boundary condition at some distance up the slope. As shown in Figure 4.2 , the situation is two solitary waves propagating symmetrically towards a submerged island between them.

We present results for two experiments with incident solitary waves of amplitudes $a_{0}=0.3,0.4$ (we used $M=8$ and $M_{b}=4$ ). In both cases, the bottom slope is fairly steep $(s=1 / 18)$, starting at $x=d=8$ and ending at $x=25$. A resolution of $N=512$ points was specified over the whole domain $0 \leq x \leq 52$. (Preliminary results have appeared in [28] for a different implementation of the DNO.) Figures 4.3 and 4.4 show the comparison of wave profiles at two advanced stages of shoaling for $a_{0}=0.3,0.4$, respectively. The BE solution exhibits a slightly sharper and higher wave crest than the spectral solution, and these discrepancies become more pronounced as the solitary waves approach breaking. It is not surprising that near the onset of breaking the BE results are more reliable, since the present method uses a Fourier spectral decomposition and requires that the free surface be represented as the graph of a function, $y=\eta(x, t)$. Nevertheless, our results are found to be close to the BE ones until near the time of breaking. In both cases $a_{0}=0.3,0.4$, the rear faces of the waves match almost perfectly and the wave crests have nearly the same locations.

4.3. Bragg reflection over sinusoidally varying topography. We now consider Bragg reflection over a sinusoidal bottom "ripple patch." This problem has 
(a)
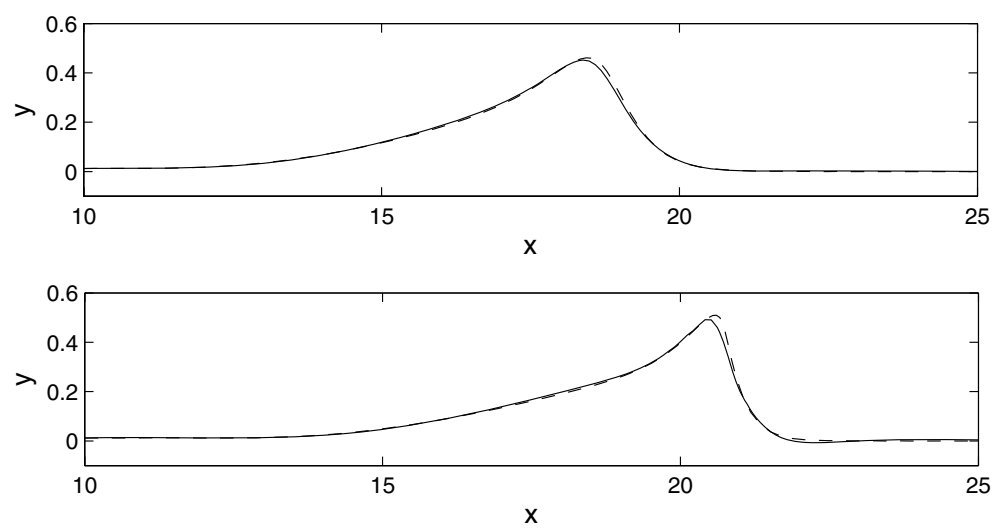

(b)

FIG. 4.4. Comparison of solitary wave profiles between boundary element (dashed line) and spectral (solid line) methods for $a_{0}=0.4$ at times (a) $t=10.2$ and (b) $t=12$.

drawn considerable attention in the literature (e.g., [36, 14, 30, 33]) because it represents a simple situation of the interaction between surface water waves and sediment transport with applications in coastal engineering. For small incident wave and bottom slopes, reflection near Bragg resonance is predicted well by multiple-scale linearized perturbation theory [36]. Here we numerically examine higher-order nonlinear effects using our spectral method. The conditions in Davis and Heathershaw [15] are used in order to compare with their experiments. The bottom "ripple patch" is defined by

$$
\beta(x)=d \sin \left(k_{b} x\right) \quad \text { for } \quad-L_{0} \leq x \leq L_{0}
$$

as depicted in Figure 4.5. The ripple slope is $k_{b} d=0.31$, the ripple amplitude is $d=0.16$, and $L_{0} / \lambda_{b}=10$ (i.e., a patch with 10 sinusoidal ripples, where $\lambda_{b}=2 \pi / k_{b}$ ).

Figure 4.6 shows the computed reflection coefficient for incident waves of steepness $k a_{0}=0.05$ and wavenumber $k$ near the (linearized) Bragg resonance condition $2 k / k_{b}=1\left(M=M_{b}=2\right)$. We use the least-squares method of Mansard and Funke [35] to evaluate the reflection coefficient from time series in the steady state. For comparison, we also show in Figure 4.6 the experimental data of Davis and Heathershaw [15] along with the linear perturbation solution obtained by Mei [36]. The downshift of the resonant peak relative to the linear value is clearly observed, which is consistent with the experimental data. This wavenumber downshift is not predicted by linear theory and can be attributed to nonlinear effects associated with the problem. Similar results were reported in $[33,4]$, where different numerical models were used.

4.4. Harmonic generation over a submerged bar. It is well known that regular waves decompose into higher-frequency free waves as they propagate past a submerged bar, as shown in experimental work $[3,17,18]$. As the waves travel up the front slope of the bar, higher harmonics are generated due to nonlinear interactions, causing the waves to steepen. These harmonics are then released as free waves on the downslope, producing an irregular pattern behind the bar. This experiment is particularly difficult to simulate because it includes nonlinear interactions and requires accurate propagation of waves in both deep and shallow water, over a wide range of depths. Therefore it has often been used as a discriminating test case for nonlinear models of surface wave propagation over a variable bottom $[17,46,4]$. Here we 


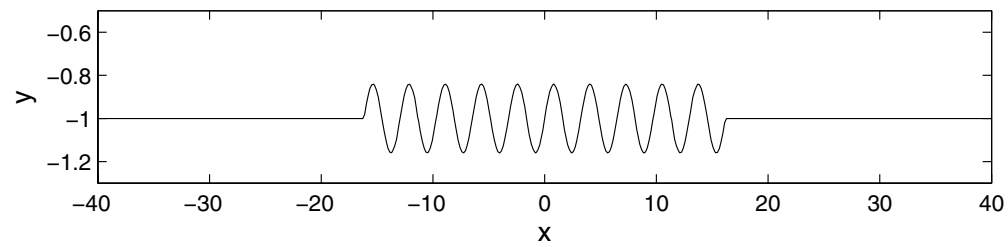

FIG. 4.5. Bottom topography with a patch of 10 sinusoidal ripples of amplitude $d=0.16$ and slope $k_{b} d=0.31$, as described in the experiments of Davis and Heathershaw [15].

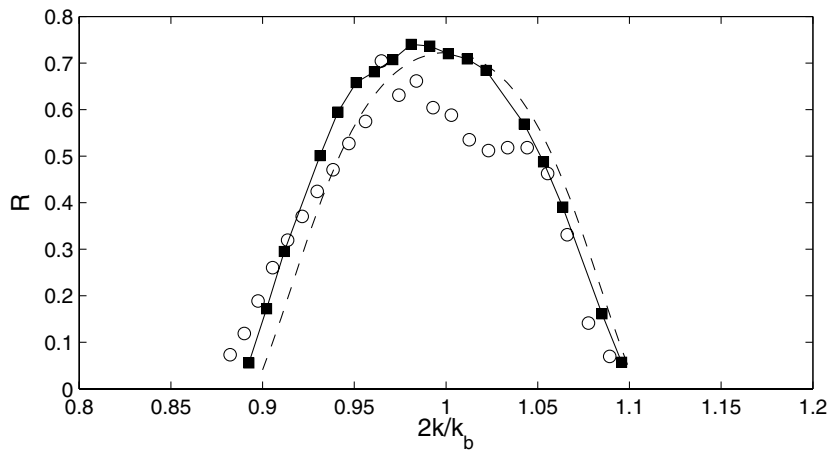

FIG. 4.6. Bragg reflection coefficient near the linearized resonance condition $2 k / k_{b}=1$ for $k a_{0}=0.05, k_{b} d=0.31, d=0.16$ : experiments (circles), linear perturbation theory (dashed line) [36], and our new numerical simulations (squares-solid line).

compare our spectral method with the experimental data of Dingemans [17]. The bottom variation is defined by

$$
\beta(x)= \begin{cases}0.05(x-6) & \text { for } 6 \leq x \leq 12 \\ 0.3 & \text { for } 12 \leq x \leq 14 \\ 0.3-0.1(x-14) & \text { for } 14 \leq x \leq 17 \\ 0 & \text { elsewhere }\end{cases}
$$

in scaled units (meters), as illustrated in Figure 4.7.

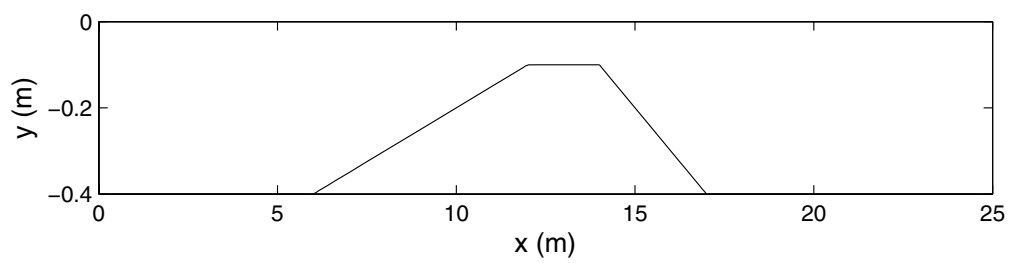

FIG. 4.7. Submerged bar for harmonic wave generation as described in the experiments of Dingemans [17].

We present numerical results for two different incident wave conditions: $\left(T_{0}, a_{0}\right)$ $=(2.02 \mathrm{~s}, 0.02 \mathrm{~m})$ and $(2.525 \mathrm{~s}, 0.029 \mathrm{~m})$, where $T_{0}$ and $a_{0}$ denote the incident wave period and height, respectively. Time histories of the surface elevations at various locations are shown in Figure 4.8. In both cases, computations were performed using

Copyright (C) by SIAM. Unauthorized reproduction of this article is prohibited. 

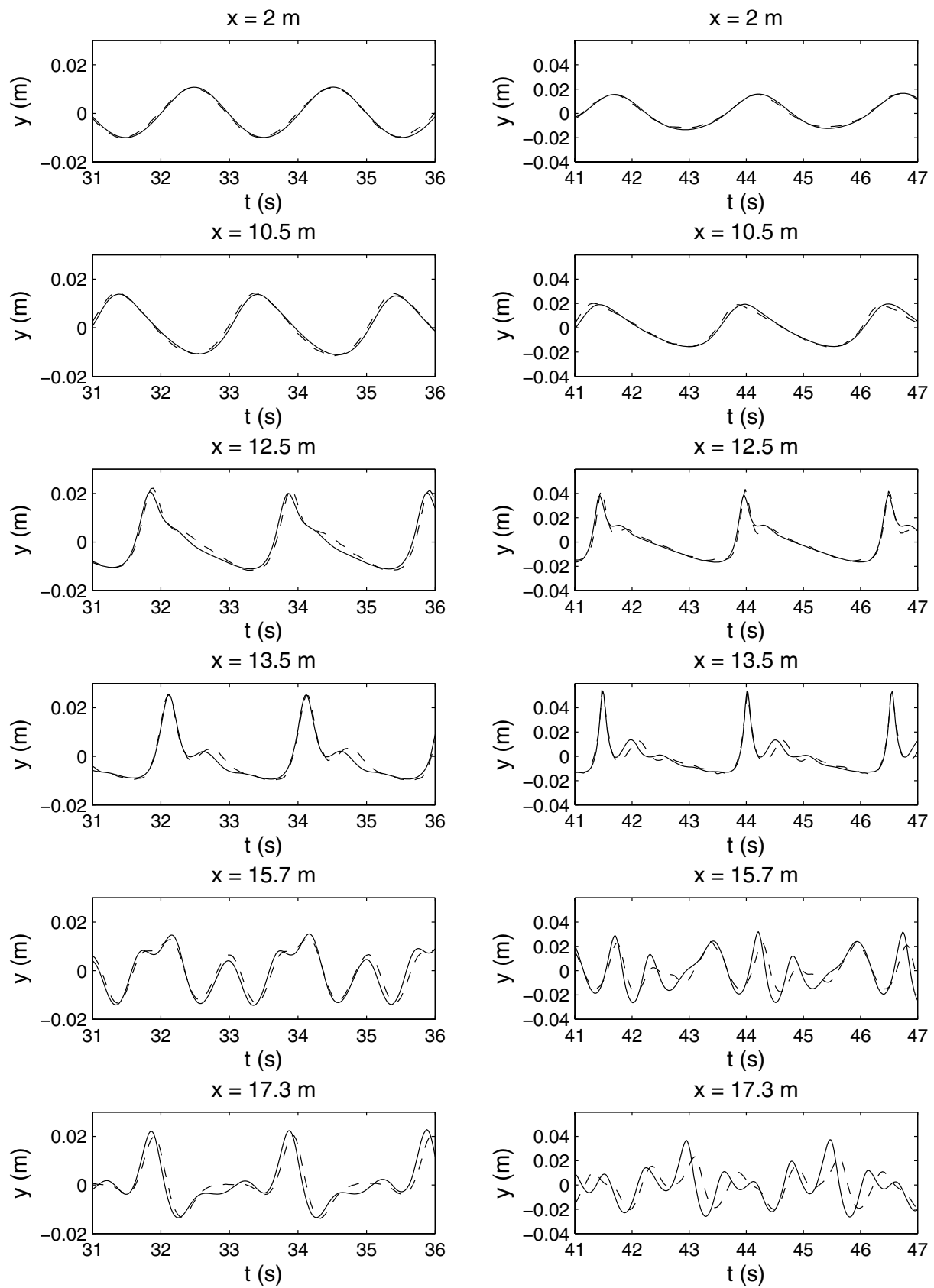

FIG. 4.8. Time series of surface elevations at various locations for waves passing over a bar: experiments (dashed line) and numerical simulations (solid line). Left column: incident wave with $T_{0}=2.02 \mathrm{~s}$ and $a_{0}=0.02 \mathrm{~m}$. Right column: incident wave with $T_{0}=2.525 \mathrm{~s}$ and $a_{0}=0.029 \mathrm{~m}$.

Copyright (C) by SIAM. Unauthorized reproduction of this article is prohibited. 
a resolution of $N=2048$ points with $M=M_{b}=8$. The time origin has been shifted so that the numerical results match the measurements for the first wave gauge at $x=2 \mathrm{~m}$. Overall, the computations compare reasonably well with the experimental data in both cases. In particular, the asymmetry of the shoaling waves and the generation of higher harmonics are reproduced well by the numerical model. Note that, in the second case (which corresponds to an incident wave of larger amplitude than in the first case), some discrepancies are observed for gauges beyond the crest of the bar (i.e., $x>13.5 \mathrm{~m}$ ). This may be explained by the occurrence of spilling breakers in the experiments as reported in [17] for this case. As we have mentioned before, due to our choice of coordinates, wave breaking is a phenomenon not permitted in the present algorithm.

4.5. Tsunami due to impulsive bottom displacement. Tsunamis are long waves of great destructive power, generated by disturbances associated primarily with earthquakes occurring at or just below the ocean floor. In deep water, a tsunami is usually of small height (only a few feet or less), but, as it reaches shallow water, its amplitude increases rapidly. Understandably, such waves have been the subject of serious attention recently.

In this final example, we consider the simple, idealized problem of a tsunami generated by the sudden uplift of a localized region of the bathymetry at $t=0$. In order to prevent instabilities in the computations, a smooth motion of the bottom is prescribed by

$$
\beta(x, t)=f(t) \beta_{0}(x),
$$

where $\beta_{0}(x)$ denotes the spatial dependence of $\beta$ and $f(t)$ is a relaxation function depicted in Figure 4.9. The relaxation time was chosen small enough $(\sim 0.1)$ to closely model an impulsive bottom displacement, and the vertical bottom velocity in this case is given by $v(x, t)=\partial_{t} \beta$.

(a)

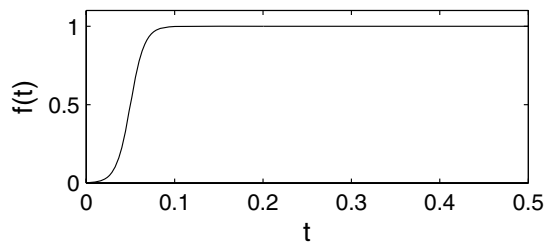

(b)

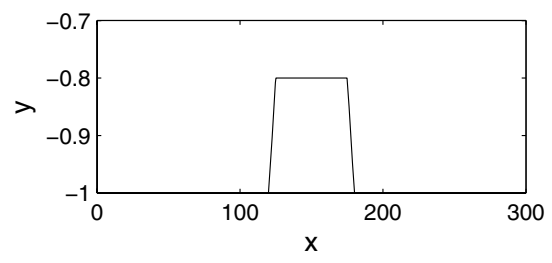

FIG. 4.9. (a) Time dependence $f(t)$ and (b) spatial dependence $-h_{0}+\beta_{0}(x)$ for the bottom geometry in the case where $\beta(x, t)=f(t) \beta_{0}(x)$, meant to simulate a tsunami-generating "earthquake."

Figure 4.10 shows the resulting wave profile at $t=100$. The simulations were run for $N=1024$ and $M=M_{b}=4$. The bottom disturbance was specified to be relatively small $\left(\left\|\beta_{0}\right\|_{\infty}=0.2\right)$ in order to be close to realistic conditions and to allow for a meaningful comparison with the linear solution [37],

$$
\eta(x, t)=\frac{1}{2 \pi} \int_{\mathbb{R}} e^{i k x} \hat{\beta}_{0}(k) \frac{\cos (\omega t)}{\cosh \left(h_{0} k\right)} d k,
$$

where $\hat{\beta}_{0}(k)$ denotes the $k$ th Fourier component of $\beta_{0}(x)$. Overall, the amplitude and profile of the tsunami computed by the spectral model are close to those predicted 


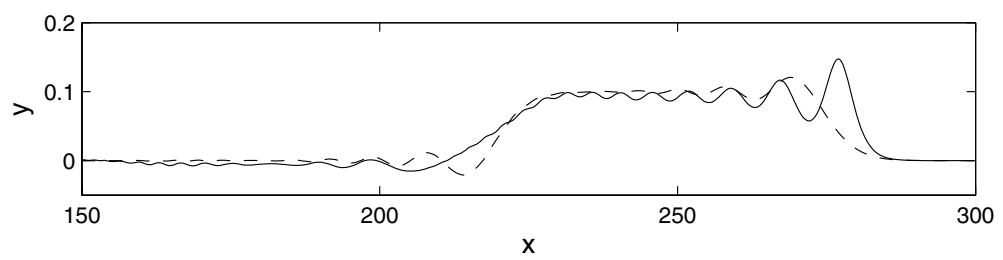

FIG. 4.10. Surface elevation (due to impulsive bottom displacement) at $t=100$ : linear (dashed line) and fully nonlinear (solid line) solutions.

by linear theory. The main difference we observe is that the leading wave in the fully nonlinear solution is larger and has progressed further compared with the linear solution. This is similar to the results obtained by Fructus and Grue [22], who used a boundary integral method to study this problem.

5. Conclusions. We have developed a numerical method to simulate nonlinear free-surface waves on an ocean of variable depth. The model is applicable to both static and moving (time-dependent) bottom topography. As an extension of the work of Craig and Sulem [13], Craig et al. [10], and Guyenne and Nicholls [28], it is based on the expansion of the DNO as a Taylor series in terms of the surface and bottom variations; however, the terms in the Taylor series have been reformulated to eliminate all reference to the "exponentially unbounded" operators which appear in [10, 28]. Spatial discretization can be efficiently performed by a pseudospectral method using the fast Fourier transform, thus allowing for the rapid evaluation of the DNO. Efficient procedures for wave generation/absorption have also been implemented to simulate nonperiodic boundary conditions.

By a series of physically relevant experiments, we have shown that the method is robust and accurate and can be applied to a wide range of problems in coastal engineering. In particular, despite the limitations inherent to the particular representation (operator expansions; see [42, 43, 44]) of the Taylor coefficients of the DNO, satisfactory results have been obtained even for fairly steep waves over highly varying bottom geometries. Finally, we emphasize that this approach is not restricted to twodimensional flows. The mathematical formulation of the method is presented here for any spatial dimension $n \geq 2$, and we envision computations of three-dimensional free-surface waves in the near future.

Acknowledgments. The authors would like to thank W. Craig, J. T. Kirby, and C. Sulem for encouragement and helpful discussions. We are grateful to J. T. Kirby, who kindly provided the technical reports of Davis and Heathershaw as well as the code to compute the Bragg reflection coefficient by the least-squares method of Mansard and Funke. We also thank M. W. Dingemans for providing the data from the Delft Hydraulics bar experiments.

\section{REFERENCES}

[1] W. J. D. Bateman, C. Swan, and P. H. Taylor, On the efficient numerical simulation of directionally spread surface water waves, J. Comput. Phys., 174 (2001), pp. 277-305.

[2] J. T. BEale, A convergent boundary integral method for three-dimensional water waves, Math. Comp., 70 (2001), pp. 977-1029.

[3] S. Beji AND J. A. BAtTJes, Experimental investigations of wave propagation over a bar, Coastal Engng., 19 (1993), pp. 151-162. 
[4] H. B. Bingham And Y. Agnon, A Fourier-Boussinesq method for nonlinear water waves, Eur. J. Mech. B Fluids, 24 (2005), pp. 255-274.

[5] A. P. Calderón, Cauchy integrals on Lipschitz curves and related operators, Proc. Nat. Acad. Sci. U.S.A., 75 (1977), pp. 1324-1327.

[6] C. Canuto, M. Y. Hussaini, A. Quarteroni, and T. Zang, Spectral Methods in Fluid Dynamics, Springer-Verlag, New York, 1988.

[7] D. Clamond, D. Fructus, J. Grue, and Ø. Kristiansen, An efficient model for threedimensional surface wave simulations. Part II: Generation and absorption, J. Comput. Phys., 205 (2005), pp. 686-705.

[8] R. CoIfman and Y. MeYer, Nonlinear harmonic analysis and analytic dependence, in Pseudodifferential Operators and Applications, AMS, Providence, RI, 1985, pp. 71-78.

[9] W. Craig, P. Guyenne, And H. Kalisch, Hamiltonian long wave expansions for free surfaces and interfaces, Comm. Pure Appl. Math., 58 (2005), pp. 1587-1641.

[10] W. Craig, P. Guyenne, D. P. Nicholls, And C. Sulem, Hamiltonian long wave expansions for water waves over a rough bottom, Proc. R. Soc. Lond. Ser. A Math. Phys. Eng. Sci., 461 (2005), pp. 839-873.

[11] W. Craig and D. P. Nicholls, Traveling two and three dimensional capillary gravity water waves, SIAM J. Math. Anal., 32 (2000), pp. 323-359.

[12] W. Craig, U. Schanz, And C. Sulem, The modulation regime of three-dimensional water waves and the Davey-Stewartson system, Ann. Inst. Henri Poincaré Anal. Non Linéaire, 14 (1997), pp. 615-667.

[13] W. Craig And C. Sulem, Numerical simulation of gravity waves, J. Comput. Phys., 108 (1993), pp. $73-83$.

[14] R. A. Dalrymple And J. T. Kirby, Water waves over ripples, J. Waterway, Port, Coast., Ocean Engng, 112 (1986), pp. 309-319.

[15] A. G. Davis And A. D. Heathershaw, Surface wave propagation over sinusoidally varying topography, J. Fluid Mech., 144 (1984), pp. 419-443.

[16] R. G. Dean and R. A. Dalrymple, Water Wave Mechanics for Engineers and Scientists, World Scientific, Singapore, 1991.

[17] M. W. Dingemans, Comparison of Computations with Boussinesq-Like Models and Laboratory Measurements, Technical report H1684.12, Delft Hydraulics, Delft, The Netherlands, 1994.

[18] M. W. Dingemans, Water Wave Propagation over Uneven Bottoms, World Scientific, Singapore, 1997.

[19] D. G. Dommermuth and D. K. P. Yue, A high-order spectral method for the study of nonlinear gravity waves, J. Fluid Mech., 184 (1987), pp. 267-288.

[20] C. Fochesato And F. Dias, A fast method for nonlinear three-dimensional free-surface waves, Proc. R. Soc. Lond. Ser. A Math. Phys. Eng. Sci., 462 (2006), pp. 2715-2735.

[21] M. Frigo And S. G. Johnson, The Fastest Fourier Transform in the West, Technical report MIT-LCS-TR-728, MIT, Cambridge, MA, 1997, http://theory.lcs.mit.edu/fftw/.

[22] D. Fructus and J. Grue, Fully nonlinear and simplified models for $3 D$ water waves generated by a moving bottom, in Proceedings of the 20th IWWWFB, Longyearbyen, Norway, 2005.

[23] D. Gottlieb and S. A. Orszag, Numerical Analysis of Spectral Methods: Theory and Applications, CBMS-NSF Regional Conf. Ser. in Appl. Math. 26, SIAM, Philadelphia, 1977.

[24] S. T. Grilli, P. Guyenne, And F. Dias, A fully nonlinear model for three-dimensional overturning waves over an arbitrary bottom, Internat. J. Numer. Methods Fluids, 35 (2001), pp. 829-867.

[25] S. T. Grilli, I. A. Svendsen, And R. Subramanya, Breaking criterion and characteristics for solitary waves on plane beaches, J. Waterway, Port, Coast., Ocean Engng., 123 (1997), pp. 102-112.

[26] R. H. J. GRImshaw, The solitary wave in water of variable depth, J. Fluid Mech., 42 (1970), pp. 639-656.

[27] P. Guyenne And S. T. GRILli, Numerical study of three-dimensional overturning waves in shallow water, J. Fluid Mech., 547 (2006), pp. 361-388.

[28] P. Guyenne And D. P. Nicholls, Numerical simulation of solitary waves on plane slopes, Math. Comput. Simulation, 69 (2005), pp. 269-281.

[29] B. Hu And D. P. Nicholls, Analyticity of Dirichlet-Neumann operators on Hölder and Lipschitz domains, SIAM J. Math. Anal., 37 (2005), pp. 302-320.

[30] J. T. KIRBY, A note on Bragg scattering of surface waves by sinusoidal bars, Phys. Fluids, 5 (1993), pp. 380-386.

[31] H. Lamb, Hydrodynamics, 6th ed., Cambridge University Press, Cambridge, UK, 1993.

[32] D. LAnnes, Well-posedness of the water-waves equations, J. Amer. Math. Soc., 18 (2005), pp. $605-654$. 
[33] Y. Liu AND D. K. P. Yue, On generalized Bragg scattering of surface waves by bottom ripples, J. Fluid Mech., 356 (1998), pp. 297-326.

[34] P. A. Madsen, D. R. Fuhrman, and B. Wang, A Boussinesq-type method for fully nonlinear waves interacting with a rapidly varying bathymetry, Coastal Engng., 53 (2006), pp. 487504.

[35] E. P. D. MANSARD AND E. R. Funke, The measurement of incident and reflected spectra using a least squares method, in Proceedings of the 17th Coastal Engineering Conference, ASCE, New York, 1980, pp. 154-172.

[36] C. C. MEI, Resonant reflection of surface water waves by periodic sandbars, J. Fluid Mech., 152 (1985), pp. 315-335.

[37] C. C. MeI, The Applied Dynamics of Ocean Surface Waves, World Scientific, Singapore, 1989.

[38] M. D. MiLDER, An improved formalism for wave scattering from rough surfaces, J. Acoust. Soc. Amer., 89 (1991), pp. 529-541.

[39] P. A. Milewski, A formulation for water waves over topography, Stud. Appl. Math., 100 (1998), pp. 95-106.

[40] A. NachBIN, A terrain-following Boussinesq system, SIAM J. Appl. Math., 63 (2003), pp. 905922.

[41] D. P. Nicholls, Traveling water waves: Spectral continuation methods with parallel implementation, J. Comput. Phys., 143 (1998), pp. 224-240.

[42] D. P. Nicholls and F. Reitich, A new approach to analyticity of Dirichlet-Neumann operators, Proc. Roy. Soc. Edinburgh Sect. A, 131 (2001), pp. 1411-1433.

[43] D. P. Nicholls and F. Reitich, Stability of high-order perturbative methods for the computation of Dirichlet-Neumann operators, J. Comput. Phys., 170 (2001), pp. 276-298.

[44] D. P. Nicholls and F. Reitich, Analytic continuation of Dirichlet-Neumann operators, Numer. Math., 94 (2003), pp. 107-146.

[45] D. P. Nicholls AND M. TABer, Joint analyticity and analytic continuation for DirichletNeumann operators on doubly perturbed domains, J. Math. Fluid Mech., to appear.

[46] R. A. Sмiтh, An operator expansion formalism for nonlinear surface waves over variable depth, J. Fluid Mech., 363 (1998), pp. 333-347.

[47] M. TANaka, The stability of solitary waves, Phys. Fluids, 29 (1986), pp. 650-655.

[48] B. J. West, K. A. Brueckner, R. S. Janda, D. M. Milder, and R. L. Milton, A new numerical method for surface hydrodynamics, J. Geophys. Res., 92 (1987), pp. 1180311824 .

[49] M. Xue, H. Xü, Y. Liu, And D. K. P. Yue, Computations of fully nonlinear three-dimensional wave-wave and wave-body interactions. I. Dynamics of steep three-dimensional waves, J. Fluid Mech., 438 (2001), pp. 11-39.

[50] V. E. ZAKHAROV, Stability of periodic waves of finite amplitude on the surface of a deep fluid, J. Appl. Mech. Tech. Phys., 9 (1968), pp. 190-194.

Copyright $@$ by SIAM. Unauthorized reproduction of this article is prohibited. 